\title{
Modelling and Analysis of Constant False Alarm Rate Performance in Presence of Jamming Environments
}

\author{
Xiaozhou Chen $(\mathbb{D}$, Mengzhong Hu $(\mathbb{D}$, and Shu Lu \\ Nanjing Changfeng Aerospace Electronic Technology Co. Ltd., Nanjing, China \\ Correspondence should be addressed to Mengzhong Hu; weibohyb@163.com
}

Received 24 October 2021; Revised 5 January 2022; Accepted 8 January 2022; Published 31 January 2022

Academic Editor: Raffaele Solimene

Copyright (c) 2022 Xiaozhou Chen et al. This is an open access article distributed under the Creative Commons Attribution License, which permits unrestricted use, distribution, and reproduction in any medium, provided the original work is properly cited.

\begin{abstract}
A novel "Bernoulli experiment model" of constant false alarm rate (CFAR) is presented in order to analyze CFAR performance in jamming condition. In this model, target detection can be treated as a sequence of independent subevents, and the detection process equals to pick up target energy from an energy pool diluted with noise, interference, and jamming pulses. In a multiplejammer case, each jammer contributes independently, and the content of the energy pool only relates to the corresponding jammer. Impacts of jammer factors, such as signal-to-interference ratio (SIR), jamming-to-interference ratio (JIR), the number of false targets in reference cells, and the number of false targets in a cell under test (CUT), are analyzed and compared in detail, and two jamming operation principles are deduced as an application. The deduction procedure and conclusions drawn from the model can also be utilized on similar occasions.
\end{abstract}

\section{Introduction}

The detection of an unknown signal needs to compare the signal plus interference power in the cell under test (CUT) to a predetermined threshold. The case that interference exceeds the threshold with no target echo presented in CUT is defined as a false alarm. In Neyman-Pearson criterion, we need to control the false alarm rate to an acceptable range while maximizing the detection probability [1]. From that perspective, estimating the interference level and dynamically adjusting the detection threshold accordingly is required. This gives birth to constant false alarm rate (CFAR) techniques.

The core idea of CFAR is to utilize some range cells lagging or leading the CUT as reference cells, design an algorithm to estimate interference from the reference cells, and determine the threshold according to the estimation. In this way, thresholds will track the changes of interference automatically, and the false alarm rate will be maintained to a constant value. The traditional CFAR scheme is cell-average (CA-) CFAR, where the estimation is based on the mean value of all reference cells. CA-CFAR performs well in homogeneous environments but suffers from self-masking and mutual target masking in heterogeneous environments. Improvements are designed by selecting or discarding a certain number of reference cells to overcome the above drawbacks, yielding greatest of (GO) [2-4], smallest of (SO) [5], trimmed mean(TM) [6, 7], and order statistic (OS) CFARs $[8,9]$. All of the above schemes concentrate on the cell selecting strategies, but the basic models and fundamental computations are like CA-CFAR.

Nowadays, sophisticated and intelligent jammers bring about unprecedented challenges for CFAR. Traditionally, jammers are able to create large amounts of false target "clouds," covering most areas on a radar screen, where real targets are entirely submerged. If the false targets are dense enough, they act like continuous wave noise, effectively raising the dynamic threshold and reducing the probability of detection. Moreover, advanced techniques and strategies such as cooperative jamming $[10,11]$, distributive jamming $[12,13]$, and smart noise $[14,15]$ make it more complicating to evaluate effects of every single technique. A clear and precise mathematical model revealing the relationship between jamming parameters and CFAR characteristics is urgently required. 
Unfortunately, compared to the deep research on CFAR techniques, CFAR performance in complex electromagnetic environment, especially in jamming conditions, is not frequently discussed. Some researchers created rough models to analyze CFAR in heterogeneous environments, by which various CFAR schemes were compared $[4,16,17]$. However, the presented model was not widely applicable and did not unveil its physical meaning. Yan-Juan et al. [18] created a mathematical model and calculated the impact of jammerto-signal ratio and distance of false targets, but the model is not sufficiently simplified, and complicated expressions concealed the direct relationship between each parameter. Bachmann et al. [19] focused on dynamic interaction between radars and jammers, and the optimization method under certain constrains was illustrated. Liu and $\mathrm{Li}$ [20] discussed the power cooperative and time cooperative jamming case, and effects from different jamming modulations were analyzed in detail. Neither Bachmann et al. [19] nor Liu and $\mathrm{Li}$ [20] presented a generalized model. Kunpeng et al. [21] deduced the CFAR detection capability under a type of coherent jamming (namely, intermittent sampling and repeated forwarding) and compared the effects of corresponding parameters. But the conclusion is unavailable in complicating random electronic environments since the deduction was not based on statistical analysis.

To overcome the above shortcomings, this paper mainly makes the following contributions:

(1) A "Bernoulli experiment model" (BEM) is constructed to compute the probability of detection of CFAR. Unlike in reference [18-20], this model provides an analytical, closed-form, and adequately simplified formation to describe the performance in jamming conditions and can be calculated directly since the parameters are determined. Similar ideas can be absorbed to utilize in the detection of slight changes in a sensor network [22].

(2) BEM is more generalized and widely applicable, and can be transformed into several special cases, where models in reference $[4,16,17]$ are only one of them. Moreover, it has a clear physical meaning, where the detection process can be seen as a sequence of Bernoulli experiments to pick the desired energy from the energy pool.

(3) Influences of the number of jamming cells, jammingto-interference ratio (JIR) and signal-to-interference ratio (SIR), are quantitatively analyzed and compared in detail with the help of the BEM. As an application, we obtain the "optimum power distribution principle" and "maximum jamming cells principle" in Section 4.4 to better understand and predict jammers' operation before finding an efficient counter measurement. Since the model is based on statistical analysis, these influences and principles are more applicable than those in reference [21].

(4) An analytic deduction is presented by means of rigorous direct integral, where the calculation techniques used in the derivation process will provide valuable inspiration for solving similar problems. Simplification using the moment generating function (MGF) simplifies and generalizes the deduction, which is also shown in Section 3.

1.1. Manuscript Notation. Italic letters denote scalar parameters, with capital letter $Z$ and lower-case letter $z$ denoting the random variable and constant variable, respectively. $P(\cdot)$ or $f(\cdot)$ denotes the probability density function (PDF) of a random variable, while $P(\cdot \mid \cdot)$ or $f(\cdot \mid \cdot)$ is the corresponding conditional counterparts. $*$ denotes the convolution operator, ! denotes factorial, and finally, $(\cdot)_{M}$ denotes rising factorials in the Pochhammer symbol.

\section{CFAR Principles}

Figure 1 depicts the brief flow of CFAR. After an inclusion of a series of signal processing but not limited to A/D converter, doppler processing, pulse compressing, and array processing, digital samples are passed to a square law detector to convert complex values into their magnitudesquared values. During this noncoherent integration process, the phase information of complex data is discarded, leading to a loss in integration gain. A certain number of data from leading and lagging cells are stored and selected according to predetermined strategies before they are added up. At last, the threshold is obtained from the product of the summation and coefficient $\alpha$, which represents our expected false alarm rate level.

The output of square law detector is a random variable. Under $H_{0}$ hypothesis (where only interference, yet no target return exists in the CUT), the PDF of output $z$ can be expressed as

$$
P_{Z}(z)=\frac{1}{\sigma_{i}^{2}} \exp \left(-\frac{z}{\sigma_{i}^{2}}\right),
$$

where $\sigma_{i}^{2}$ is the average interference power. Interference originates from unwanted clutter, scatter, diffraction, and thermal noise (we do not exclude thermal noise from interference, since they are treated as a whole in the following analysis). Here, we assume that the interference is homogeneous; i.e., the interference is independent identically distributed in both leading, lagging reference cells, and CUT, which is restrictive especially in mountain or urban backgrounds. The nonhomogeneous scenario can be discussed according to the main idea from reference [23]. For distributed multiradar systems, the detection performance in nonstationary Weibull distributed clutter is discussed from reference [24].

Assuming that $N$ reference cells are selected according to specific strategy and each has the same probability density as equation (1), the joint PDF of interference power is the product of each PDF, thereby

$$
P_{Z}(z)=\frac{1}{\left(\sigma_{i}^{2}\right)^{N}} \exp \left(\frac{-\sum_{n=1}^{N} z_{n}}{\sigma_{i}^{2}}\right),
$$




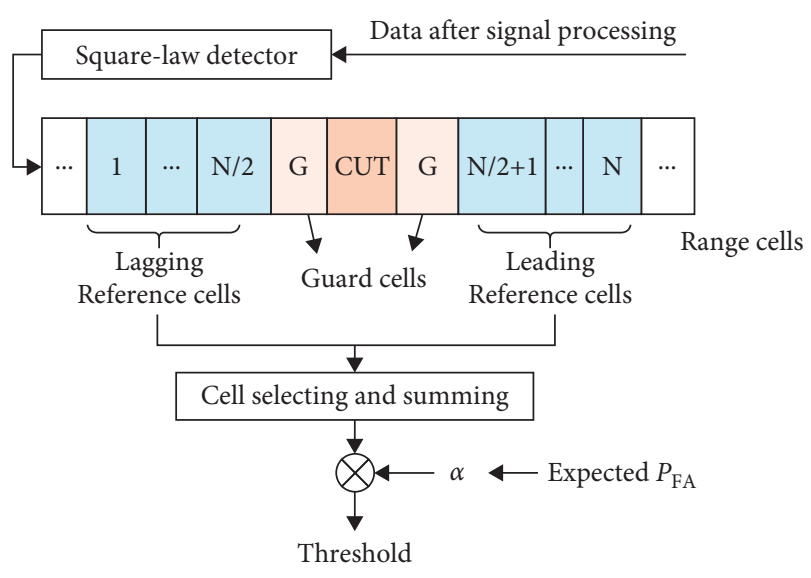

FIgURE 1: Basic principle of CFAR.

where $z=\left\{z_{1}, \ldots, z_{N}\right\}$. Then, the maximum likelihood estimate of average interference power in all the reference cells is

$$
\widehat{\sigma}_{i}^{2}=\frac{1}{N} \sum_{n=1}^{N} z_{n}
$$

In CFAR, the threshold $U_{\mathrm{T}}$ is defined as the product of $\widehat{\sigma}_{i}^{2}$ and the constant coefficient $\alpha$; thus,

$$
U_{T}=\alpha \widehat{\sigma}_{i}^{2},
$$

where $\alpha$ is determined by desired false-alarm probability $P_{\mathrm{FA}}$ :

$$
\alpha=N\left(P_{\mathrm{FA}}^{-1 / N}-1\right) .
$$

Given the Swerling 1 or 2 target PDF $f_{1}\left(x \mid H_{1}\right)$ under $H_{1}$ hypothesis (where CUT contains interference and target return) is still exponentially distributed similar to equation $(1)$, and $\sigma_{i}^{2}$ is replaced with total power of interference and target $\left(\sigma_{i}^{2}+\sigma_{t}^{2}\right)$; therefore,

$$
f_{\text {CUT }}\left(x \mid H_{1}\right)= \begin{cases}\frac{1}{\sigma_{i}^{2}+\sigma_{t}^{2}} \exp \left(-\frac{x}{\sigma_{i}^{2}+\sigma_{t}^{2}}\right), & x \geq 0, \\ 0, & x<0 .\end{cases}
$$

Without losing generosity, we induce $\gamma_{\text {CUT }}$ as $\alpha$ times inverse of total power of interference $\sigma_{i}^{2}$ and target $\sigma_{t}^{2}$, namely,

$$
\frac{\gamma_{\mathrm{CUT}}}{\alpha}=\left(\sigma_{i}^{2}+\sigma_{t}^{2}\right)^{-1}=\left[\sigma_{i}^{2}(1+\eta)\right]^{-1} .
$$

Here, $\eta \triangleq \sigma_{t}^{2} / \sigma_{i}^{2}$ is defined as SIR.

The probability of detection can be calculated as the integral from $U_{T}$ to infinity.

$$
P_{\mathrm{D}}=\int_{U_{T}}^{\infty} f_{\mathrm{CUT}}\left(x \mid H_{1}\right) \mathrm{d} x .
$$

Substituting equation (6) into (8), we obtain that

$$
\begin{aligned}
P_{\mathrm{D}}\left(\gamma_{\mathrm{CUT}} \mid \widehat{\sigma}_{i}^{2}\right) & =\int_{\alpha \widehat{\sigma}_{i}^{2}}^{\infty} \frac{\gamma_{\mathrm{CUT}}}{\alpha} \exp \left(-\frac{\gamma_{\mathrm{CUT}}}{\alpha} x\right) \mathrm{d} x \\
& =\exp \left(-\gamma_{\mathrm{CUT}} \cdot \widehat{\sigma}_{i}^{2}\right) .
\end{aligned}
$$

Given the dictated $P_{\mathrm{FA}}, P_{\mathrm{D}}$ can be derived once $\widehat{\sigma}_{i}^{2}$ is determined. Considering $\widehat{\sigma}_{i}^{2}$ is a random variable, the expected value of $P_{\mathrm{D}}$ is

$$
\overline{P_{\mathrm{D}}}\left(\gamma_{\mathrm{CUT}}\right)=\int_{0}^{\infty} P_{\mathrm{D}}\left(\gamma_{\mathrm{CUT}} \mid \widehat{\sigma}_{i}^{2}\right) f_{\widehat{\sigma}_{i}^{2}}\left(\widehat{\sigma}_{i}^{2}\right) \mathrm{d} \widehat{\sigma}_{i}^{2},
$$

where $f_{\widehat{\sigma}^{2}}\left(\widehat{\sigma}_{i}^{2}\right)$ denotes PDF of total interference power in $N$ reference cells.

\section{Bernoulli Experiment Model}

3.1. CFAR on False Targets. False targets will have great impacts on $P_{\mathrm{D}}$ and $P_{\mathrm{FA}}$, and drastically degrade CFAR performance. On the one hand, false targets in reference cells will raise the estimated interference level as well as dynamic threshold, yielding the declination of $P_{\mathrm{D}}$; on the other hand, jamming pulses in CUT will cause false alarm and increase $P_{\mathrm{FA}}$.

Assuming there is only one jammer source, radiating $M$ false points in reference cell (therefore $M$ is defined as the number of jamming cells) and $Q$ in CUT, respectively. Since the interference power in CUT has thus been lift up to $Q$ times, $\gamma_{\text {CUT }}$ (denoting as $\gamma$ ) in equation (7) can be rewritten as

$$
\begin{aligned}
\gamma & \triangleq \alpha\left(Q \sigma_{\mathrm{i}}^{2}+\sigma_{\mathrm{t}}^{2}\right)^{-1} \\
& =\alpha\left[\sigma_{i}^{2}(\mathrm{Q}+\eta)\right]^{-1} .
\end{aligned}
$$

Accordingly, $P_{\mathrm{D}}$ is transformed from equation (9) as

$$
P_{\mathrm{D}}(\gamma \mid x)=\exp (-\gamma x)
$$

letting $x$ to be the total power in reference cells.

The expectation of detection probability $\overline{P_{\mathrm{D}}}$ can be derived once $\gamma_{\text {CUT }}$ and $f_{\widehat{\sigma}^{2}}\left(\widehat{\sigma}_{i}^{2}\right)$ in equation (10) are known. Since the former is expressed in equation (11), the key step is the deduction of $f_{\widehat{\sigma}^{2}}\left(\widehat{\sigma}_{i}^{2}\right)$. Here, we directly provide the final result, and corresponding derivation is listed in Appendix if readers are interested.

$$
\overline{P_{\mathrm{D}}}=\left(1+\frac{\alpha}{N(Q+\eta)}\right)^{-N}\left(1+\frac{1}{\theta} \frac{\alpha}{N(Q+\eta)}\right)^{-M} \triangleq P_{0}^{N} P_{1}^{M},
$$

where $1 / \theta=$ JIR denotes the jammer-to-interference ratio.

As shown in equation (13), $\overline{P_{\mathrm{D}}}$ is composed of two factors, the former $P_{0}^{N}=(1+\alpha / N(Q+\eta))^{-N}$ is mainly determined by interference, and the latter $P_{1}^{M}=(1+(1 / \theta)(\alpha / N(Q+\eta)))^{-M}$ reflects the jamming effects. The number of jamming cells $M$ and JIR (denoted by $1 / \theta)$ are the main factors that influence CFAR performance. Moreover, $M$ is more influential than JIR because of its exponential effects. Comparatively, $Q$ impacts both factors, since jamming pulses in CUT become the "targets" to be detected. 
The corresponding false alarm rate $\overline{P_{\mathrm{FA}}}$ can be derived by letting $\eta=0$, namely,

$$
\overline{P_{\mathrm{FA}}}=\left(1+\frac{\alpha}{N Q}\right)^{-N}\left(1+\frac{1}{\theta} \frac{\alpha}{N Q}\right)^{-M}
$$

The above equation illustrates that, under the jamming condition, $\overline{P_{\mathrm{FA}}}$ is no more a constant value. Apparently, $\overline{P_{\mathrm{FA}}}$ will decrease when JIR or $M$ increases, inasmuch as rising power in reference cells also raises the threshold. Nevertheless, $Q$ has an opposite effect, since the growing number of false targets in CUT are more likely to be detected mistakenly.

3.2. CFAR on Noise Jamming. Noise jamming scenario can be treated as a special case of false target, where the number of jamming cells $M$ is the same as the total number of reference cells $N$. In fact, as a generalized model, equation (13) can be simplified to some expressions to adapt to concrete special occasion; for example, the steps are as follows:

(i) No jamming case. When setting $M=0$ and $Q=1$, we can get the theoretical $P_{\mathrm{D}}$ of CFAR, namely,

$$
\overline{P_{\mathrm{D} 0}}=\left(1+\frac{\alpha}{N(1+\eta)}\right)^{-N} \text {. }
$$

(ii) Multitarget case. The average of jamming power equals to that of interference; thus $\theta=1 / \eta$, and $Q=1$; therefore,

$$
\overline{P_{\mathrm{D}-M T}}=\left(1+\frac{\alpha}{N(1+\eta)}\right)^{-N}\left(1+\frac{\alpha \eta}{N(1+\eta)}\right)^{-M} \text {. }
$$

(iii) Continuous wave jamming (or noise jamming) case. In this case, jamming signal "fills up" all the reference cells, so $M=N$ and $Q=1$, namely,

$$
\overline{P_{\mathrm{D}-C W J}}=\overline{P_{\mathrm{D} 0}}\left(1+\frac{1}{\theta} \frac{\alpha}{N(1+\eta)}\right)^{-N} .
$$

As for jamming waveform, a continuous wave requires much less JIR than discrete pulses. In Section 4.3, we will see that even the slightest JIR would produce considerable effects on CFAR.

3.3. Physical Explanation. The framework of equation (13) can be interpreted from two levels: level I is from a subevent perspective (i.e., the explanation of $P_{0}$ or $P_{1}$ ), and level II is how the subevents are combined. The former is discussed in Section 3.3.1, where each subevent reflects the probability of picking the "effective target energy" from an "energy pool." The latter is analyzed in Section 3.3.2, where the total probability is the product of this subprobability, which means each subevent is mutually independent, and the results can also be extended to multiple jammer scenarios.
Deepening the research on the physical meaning may provide us with an intuitive understanding of CFAR scheme.

3.3.1. Energy Pool Model of Subevents. Noting that factors $P_{0}$ and $P_{1}$ in equation (13) can be transformed as

$$
\begin{aligned}
P_{0} & =\left(1+\frac{P_{\mathrm{FA} 0}^{-1 / N}-1}{Q+\sigma_{t 0}^{2} / \sigma_{i 0}^{2}}\right)^{-1} \\
& =\frac{\sigma_{t 0}^{2}+Q \sigma_{i 0}^{2}}{\sigma_{t 0}^{2}+Q \sigma_{i 0}^{2}+\left(P_{\mathrm{FA} 0}^{-1 / N}-1\right) \sigma_{i 0}^{2}}, \\
P_{1} & =\left(1+\frac{\sigma_{j 0}^{2}}{\sigma_{i 0}^{2}} \cdot \frac{P_{\mathrm{FA} 0}^{-1 / N}-1}{\mathrm{Q}+\sigma_{t 0}^{2} / \sigma_{i 0}^{2}}\right)^{-1} \\
& =\frac{\sigma_{t 0}^{2}+\mathrm{Q} \sigma_{i 0}^{2}}{\sigma_{t 0}^{2}+Q \sigma_{i 0}^{2}+\left(P_{\mathrm{FA} 0}^{-1 / N}-1\right) \sigma_{j 0}^{2}},
\end{aligned}
$$

where $\sigma_{t 0}^{2}, \sigma_{i 0}^{2}, \sigma_{j 0}^{2}$ refers to the average power in each cell of target return, interference, and jamming, respectively.

Equation (18) can be interpreted as the following "energy pool model." Considering there are two colors of balls uniformly merged in a pool. Red balls represent effective target power, and white balls represent effective interference power. The number of two colors of balls is $\sigma_{t 0}^{2}+Q \sigma_{i 0}^{2}$ and $\left(P_{\mathrm{FA} 0}^{-1 / N}-1\right) \sigma_{i 0}^{2}$, respectively. To detect targets is equivalent to picking up "red balls" from the pool, or more precisely, to select "effective target energy" from the "energy pool," and the probability is apparently described as equation (18) (Figure 2(a)). Equation (19) has a similar interpretation, with interference energy replaced by jamming energy (see Figure 2(b)). From this perspective, the detection process can be seen as a sequence of subevents concatenating one another (as depicted in Figure 3), and the number of subevents equals the number of cells containing interference/ jamming signals (namely, $N$ or $M$ ). Only if all the subevents happen, targets can be detected.

3.3.2. Bernoulli Experiment Model. Equation (13) implicitly indicates that, the event that signal power in CUT exceeding detection threshold can be partitioned into 2 classes of independent events: $N$ subevents with the probability mainly related to interference impact and $M$ sub-events mainly affected by jamming source. From statistical perspective, this can be named as "Bernoulli experiment model," since interference and jamming source act independently. Inspired by the intuition above, the Bernoulli experiment model can be an extent to a multijammer case.

Theorem 1. If there are $S$ jamming sources, each radiating $M_{n}$ false targets in reference cell and $Q_{n}$ in CUT, with JIR equal to $1 / \theta_{n}(n=1,2, \ldots)$, then the overall detection probability of CFAR is

$$
P_{\mathrm{D}}=\prod_{n=0}^{S}\left(1+\frac{1}{\theta_{n}} \frac{\alpha}{N(Q+\eta)}\right)^{-M_{n}},
$$






(a)



(b)

Figure 2: Energy pool model of subevents. (a) Target detection from interference. (b) Target detection from jamming.

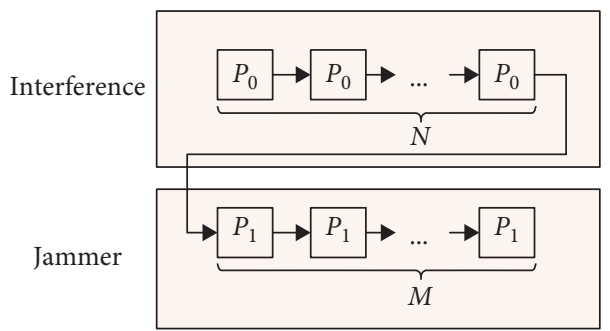

FIGURE 3: Sequence of sub-event explanation of (13).

where $n=0$ denotes the interference without jamming, with the definition $\theta_{0} \triangleq 1, M_{0} \triangleq N$, and

$$
Q=\sum_{i=1}^{S} Q_{i}
$$

Proof. As for the $n$th jamming source, the overall power of $M_{n}$ false targets has Erlang distribution similar to (A.1), by which the moment generating function (MGF) is

$$
\begin{aligned}
F_{n}(t) & =\int_{-\infty}^{\infty} f_{n}(x) e^{-t x} \mathrm{~d} t \\
& =\int_{0}^{\infty} \frac{\lambda_{n}^{M_{n}} x^{M_{n}-1}}{\left(M_{n}-1\right) !} e^{-\left(\lambda_{n}+t\right) x} \mathrm{~d} t=\left(1+\frac{t}{\lambda_{n}}\right)^{-M_{n}},
\end{aligned}
$$

where the parameter $\lambda_{n}$ is defined as the inverse of average jamming power in $M_{n}$ cells.

$$
\lambda_{n}=\frac{M_{n}}{\sigma_{n}^{2}}
$$

As shown above, the total PDF of jamming plus interference power is the convolution of each PDF, where MGF (denoted as $F(t)$ ) is the product of them.

$$
\begin{aligned}
& f(x)=f_{0}(x) * f_{1}(x) \ldots * f_{S}(x), \\
& F(t)=\prod_{n=0}^{S} F_{n}(t) .
\end{aligned}
$$

In the multijammer case, equations (11) and (12) still hold when $Q$ is redefined as equation (21). By substituting equation (12) into (10), we attain that,

$$
\overline{P_{\mathrm{D}}}=\int_{0}^{\infty} \exp (-\gamma x) f(x) \mathrm{d} x=\left.F(t)\right|_{t=\gamma} .
$$

Since

$$
\begin{aligned}
F_{n}(\gamma) & =\left(1+\frac{\gamma}{\lambda_{n}}\right)^{-M_{n}} \\
& =\left(1+\frac{\sigma_{n}^{2}}{M_{n}} \frac{\alpha}{\sigma_{i}^{2}(Q+\eta)}\right)^{-M_{n}} \\
& =\left(1+\frac{\sigma_{n}^{2} / M_{n}}{\sigma_{i}^{2} / N} \frac{\alpha}{N(Q+\eta)}\right)^{-M_{n}} \\
& =\left(1+\frac{1}{\theta_{n}} \frac{\alpha}{N(Q+\eta)}\right)^{-M_{n}} .
\end{aligned}
$$


The final solution of equation (20) can be attained by substituting equation (27) into (25). QED.

Equation (20) is not a coincidence, and there are two conditions leading to the multiplication form: (i) the prior probability of detection $P_{\mathrm{D}}(\gamma \mid x)$ can be expressed in the form of exponential function such as equation (12); (ii) the jamming/interference signals out of square law detector are exponentially distributed, and the summation has Erlang distribution. Both conditions can easily be met in reality; therefore, the independence test model is widely applicable.

In the multijammer case, the framework in Figure 3 can be extended as Figure 4. Equation (20) demonstrates that effects of different jamming sources work independently and can be analyzed, respectively. It should be noted that the number of jammer source is mathematical rather than the physical concept. Coherent jammers where pulses are from different transmitters are radiated at the same time, with energy synthesized via spatial power combining technique, can be treated as one jammer with doubled JIR; while multifunctional jammers which have different working mode (or function) are supposed to be divided into several unique monofunctional jammer sources.

\section{Numerical Analysis}

In this section, several numerical experiments are presented to examine the quantitative impacts of jamming parameters on CFAR performance. There are mainly three factors which include thepower of target echo (ascribing to SIR), jamming power in CUT (ascribing to Q), and jamming power in reference cells (ascribing to $M$ and JIR). $P_{\mathrm{D}}$ or $P_{\mathrm{FA}}$ is computed through equation (13) or (14) with parameters listed in Table 1, respectively (here, we take SIR and JIR instead of $\eta$ and $1 / \theta$ for convenience).

4.1. Impact of SIR. We depicted the relationship of $P_{\mathrm{D}}$ and SIR (or $\eta$ in the above equations) in Figure 5. The increase in SIR corresponding to an increase in $P_{\mathrm{D}}$ is intuitive, since stronger target return has more possibility to exceed threshold.

Nevertheless, once jamming power gets into the reference cell, a loss in $P_{\mathrm{D}}$ is produced. The more cells are plunged by jamming power, or equivalently more JIRs are used as the worse performance in detection. We need to take measures to raise SIR to "combat," or to some extent, to "compensate" effects of jamming cells. As shown in the curves of $M=0$ and $M=1$, we need to improve SIR to about $4 \mathrm{~dB}$ to achieve the corresponding $P_{\mathrm{D}}$ as the no-jamming case. And as $M$ increases to 5 , the compensation would be as high as $10 \mathrm{~dB}$.

4.2. Impact of $Q$. $Q$ is the number of those points, originated by jammer, just positioned in CUT, and mistakenly identified as targets. Physically, those points tend to raise the interference level in CUT, so $P_{\mathrm{D}}$ and $P_{\mathrm{FA}}$ will rise simultaneously as $Q$ increases. The impact is described in Figure 6.

Although both $P_{\mathrm{D}}$ and $P_{\mathrm{FA}}$ are positively influenced by the value of $Q$, the extents are quite different. Compared to the very limited improvement on $P_{\mathrm{D}}$ ( $P_{\mathrm{D}}$ 's improvement is no more than 0.003 for every increase in $Q$, and curves in Figure 6(a) almost overlap), it has notable effects on $P_{\mathrm{FA}}$ (tens of dozens of dB's exacerbation for every increase in $Q$ ). That is to say, once jamming pulses enter CUT, $P_{\mathrm{FA}}$ would be raised to a great extent.

4.3. Impact of $M$ and JIR. The impact of $M$ and JIR is depicted in Figure 7. Evidently, the rising of both parameters will exacerbate CFAR's detect capability, and either parameter may determine the $P_{\mathrm{D}}$ 's reduction speed with respect to the other parameter. Moreover, $M$ has relatively greater impact on $P_{\mathrm{D}}$ than JIR since lines in Figure 7(b) are more concave than those in Figure $7(\mathrm{a})$. When $M=20$ (implying all the reference cells are padded by jamming signals), merely $5 \mathrm{~dB}$ JIR may cause $P_{\mathrm{D}}$ to reduce from 0.82 to 0.44 , almost by half. From this perspective, continuous wave jammer (usually in the form of smart noise or coherent noise) would be especially destructive without much JIR.

4.4. Jamming Principle. As radars confronting with overheating challenges nowadays, sophisticated technologies enable jammers to produce a complicated waveform and comprehensive electronic attacks. Before finding an efficient way to counter them, we need to shift our eyes from radar to jammers' operation on the top of which lies in the optimum power distribution problems.

4.4.1. Optimum Jamming Power Distribution Principle. Advanced jammers usually produce hybrid signals combined with a dense false target (mode I) and narrow-band continuous wave noise (mode II) in order to raise $P_{\mathrm{FA}}$ and decrease $P_{\mathrm{D}}$ simultaneously. A common problem for an electronic warfare engineer is given as total jamming power $\sigma_{\Sigma}^{2}$ of how to distribute it to different jammer modes in order to minimize $P_{\mathrm{D}}$.

We can distinguish respective effects from each mode with the help of equation (20). Assuming power distributed to dense false target mode is $r \sigma_{\Sigma}^{2}(0<r<1)$, and to continuous noise mode is $(1-r) \sigma_{\Sigma}^{2}$. Considering false targets occupying $M$ reference cells, the JIR for each mode is $r \sigma_{\Sigma}^{2} / M \sigma_{i}^{2}$ and $(1-r) \sigma_{\Sigma}^{2} / N \sigma_{i}^{2}$, respectively. Then, $P_{\mathrm{D}}$ under this complex jammer condition is

$$
P_{\mathrm{D}}=P_{\mathrm{D} 0}\left(1+\frac{r \sigma_{\Sigma}^{2}}{M \sigma_{i}^{2}} \beta\right)^{-M}\left(1+\frac{(1-r) \sigma_{\Sigma}^{2}}{N \sigma_{i}^{2}} \beta\right)^{-N},
$$

where $\beta \triangleq k-1=\alpha /(N(Q+\eta))$. Equation (28) can be written in a logarithmic way as

$$
\ln P_{\mathrm{D}}=\ln P_{\mathrm{D} 0}-M \ln \left(1+\frac{r \sigma_{\Sigma}^{2}}{M \sigma_{i}^{2}} \beta\right)-N \ln \left(1+\frac{(1-r) \sigma_{\Sigma}^{2}}{N \sigma_{i}^{2}} \beta\right)
$$

Letting the derivation of $\ln P_{\mathrm{D}}$ with respect to $r$ equal to 0 , we can get 


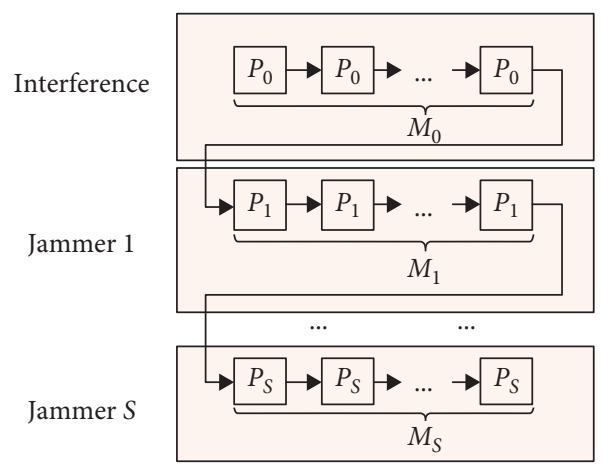

FIGURE 4: Framework of Bernoulli experiment model.

Table 1: Parameter setting.

\begin{tabular}{|c|c|c|c|c|}
\hline Section & SIR $(\mathrm{dB})$ & $M$ & $\operatorname{JIR}(\mathrm{dB})$ & Q \\
\hline 4.1 & $0 \sim 30$ & $0 / 1 / 5 / 15$ & 15 & 1 \\
\hline 4.2 & 20 & $0 \sim 10$ & 15 & $1 / 3 / 5 / 7$ \\
\hline $4.3(\mathrm{a})$ & 20 & $1 / 5 / 10 / 20$ & $-5 \sim 20$ & 1 \\
\hline $4.3(\mathrm{~b})$ & 20 & $0 \sim 20$ & $5 / 10 / 15 / 20$ & 1 \\
\hline General setting & \multicolumn{4}{|c|}{$P_{\mathrm{FA} 0}=10^{-6}, \alpha=19.91, N=20$} \\
\hline
\end{tabular}

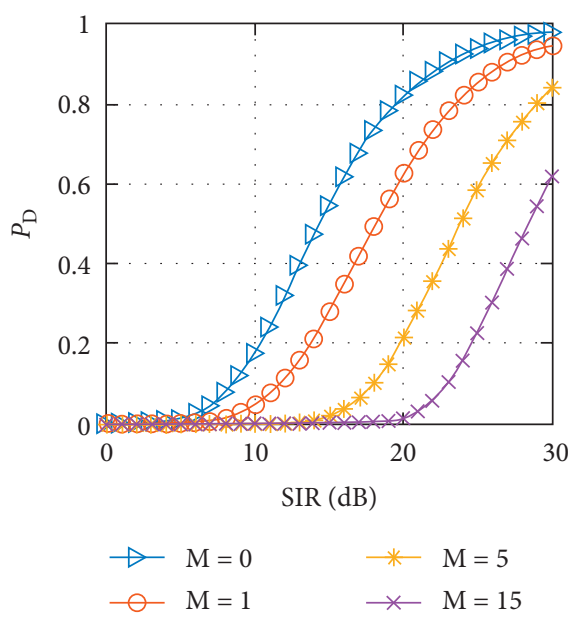

FIGURE 5: Impact of SIR on $P_{\mathrm{D}}$.

$\frac{\mathrm{d} \ln P_{\mathrm{D}}}{\mathrm{d} r}=-M \frac{\sigma_{\Sigma}^{2} / M \sigma_{i}^{2} \beta}{1+r \sigma_{\Sigma}^{2} / M \sigma_{i}^{2} \beta}+N \frac{\sigma_{\Sigma}^{2} / N \sigma_{i}^{2} \beta}{1+(1-r) \sigma_{\Sigma}^{2} / N \sigma_{i}^{2} \beta}=0$.

namely,

$$
r=\frac{M}{M+N}
$$

In order to reduce $P_{\mathrm{D}}$, the optimal power ratio of noise jamming and false target jamming should be $M / N$.

4.4.2. Maximum Jamming Cell Principle. Jammers usually have tunable false target density. If this parameter increases, $M$ and $Q$ would both rise, which will lead to a remarkable drop in $P_{\mathrm{D}}$ and increase in $P_{\mathrm{FA}}$.

On the one hand, from the detection perspective, jamming power will produce stronger effects, when it is evenly distributed to as many reference cells as possible, rather than just concentrating in one or a few reference cells. That can be referred to as "maximum jamming cells principle."

This interesting fact can be observed from Figure 7. Noting $P_{\mathrm{D}}(\mathrm{JIR}=20 \mathrm{~dB}, M=2)=0.21$ is much larger than $P_{\mathrm{D}}(\mathrm{JIR}=10 \mathrm{~dB}, M=20)=0.13$, and the discrepancy can be as high as $61.5 \%$. Apparently, the more dispersive the jamming energy is, the more obvious deterioration happens in $P_{\mathrm{D}}$.

It is not difficult to understand via the "Bernoulli experiment model." The distribution of jamming power to $M$ cells would create $M$ subevents and $M$ "compound reduction" in $P_{\mathrm{D}}$, which usually descends faster than the "single test."

On the other hand, the increase in $Q$ will raise the false alarm rate, but more $M$ will counteract this effect (see Figure 6(b)). A compromise should be made between those 


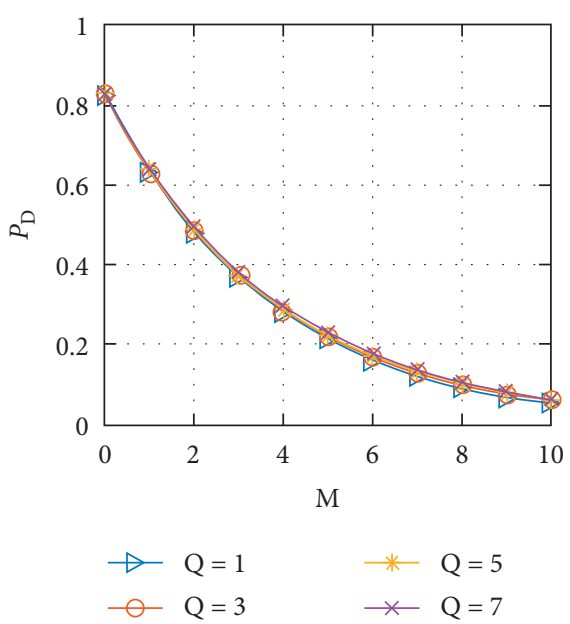

(a)

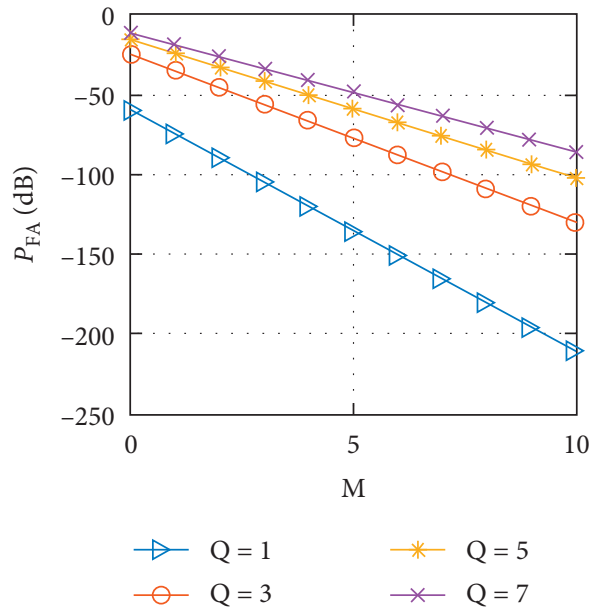

(b)

Figure 6: Impact of Q. (a) $P_{\mathrm{D}}$ vs $Q$; (b) $P_{\mathrm{FA}}$ vs $Q$.

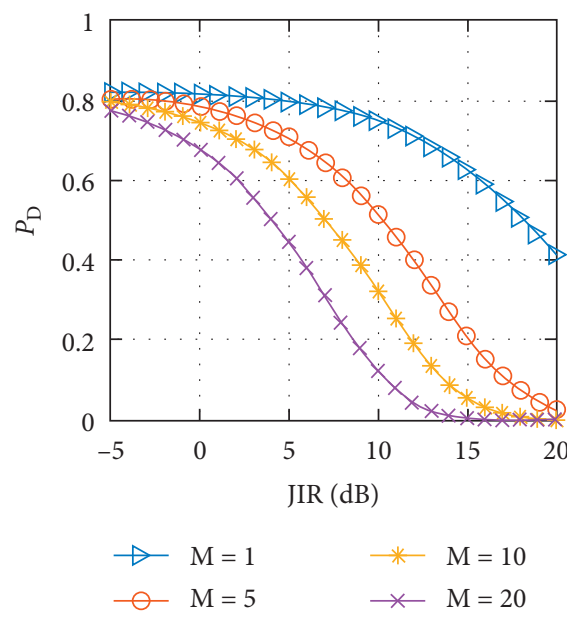

(a)

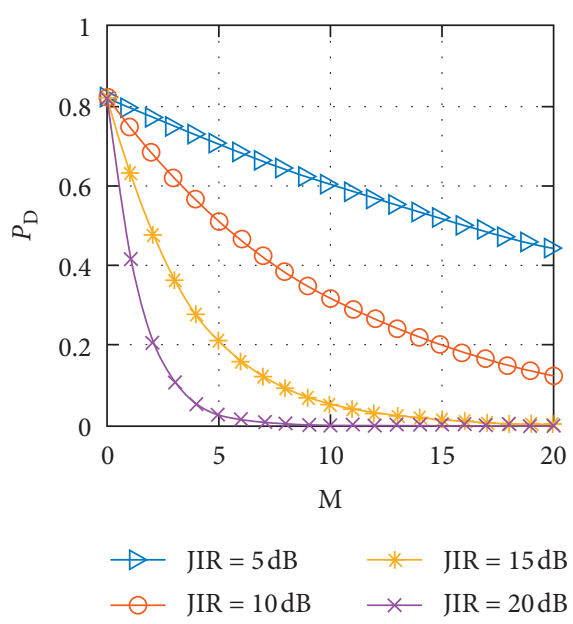

(b)

Figure 7: Impact of jammer on $P_{\mathrm{D}}$. (a) $P_{\mathrm{D}}$ vs JIR; (b) $P_{\mathrm{D}}$ vs $M$.

two factors. In order to fully quantify jamming effects, a comprehensive metric Jamming effect factor (JEF) can be defined as

$$
\mathrm{JEF} \triangleq \frac{\overline{P_{\mathrm{D}}}}{\overline{P_{\mathrm{D} 0}}}+\mu \kappa \frac{\overline{P_{\mathrm{FA} 0}}}{\overline{P_{\mathrm{FA}}}}
$$

where the subscript " 0 " means parameters without jamming, and $\kappa$ is a scaling factor to balance the scale of improvements on $\overline{P_{\mathrm{D}}}$ and $\overline{P_{\mathrm{FA}}}$. The higher JEF value, the more influences jamming have on CFAR. $\mu$ is a weight coefficient to judge the significance of $\overline{P_{\mathrm{D}}}$ and $\overline{P_{\mathrm{FA}}}$. Generally, $\mu=1$, but in some protective weapons where $\overline{P_{\mathrm{D}}}$ is primarily advocated, $\mu$ is supposed to be less than 1 , whereas in remote early warning devices where $\overline{P_{\mathrm{FA}}}$ should be rigorously controlled to a restricted range, we can set $\mu>1$. To summarize, here lists steps to find optimum parameters for jamming CFAR.

Step 1: To give the initial value of false target density
Step 2: To estimate $M$ and $Q$ according to false target density

Step 3: To compute $\overline{P_{\mathrm{D}}}$ and $\overline{P_{\mathrm{FA}}}$ through equations (13) and (14)

Step 4: To compute JEF from equation (32) and adjust false target density

Step 5: Change the value of false target density and redo Step 1 4 until JEF convergents to its maximum value

\section{Conclusion}

This paper presents a Bernoulli experiment model to quantitatively describe CFAR performance under the jamming condition. The detection process is modeled as a Bernoulli experiment with a success probability that has a clear physical essence from energy perspective. Different jammers act independently, and effects of multifunctional 
jammers can be seen as overall effects of several single jammers. BEM is a generalized model and can be transformed into specific occasions.

There exist four factors: SIR, $Q, M$, and JIR that greatly impact CFAR performance. Once jamming pulses enter the reference cells, an improvement in SIR is required to compensate the deterioration. Jamming pulses in CUT will greatly improve the probability of the false alarm to dozens of decibels. Continuous noise jamming is destructive to CFAR with relatively lower JIR.

As applications of BEM, two jamming principles are obtained in terms of power distribution of comprehensive multimode jammers and parameters setting principles, where limited power should be rationally distributed to working modes and reference cells.

It is recommended that further research be undertaken in the following areas: (1) the joint impacts of jamming and heterogeneous clutter to CFAR performance; (2) detection capabilities and comparisons of different CFAR techniques in jamming environment; (3) the quantitative effectiveness of electronic counter-counter measurements from detection perspective, etc.

\section{Appendix}

The derivation of expectation of detection probability $\overline{P_{\mathrm{D}}}$ in equation (13).
Letting $X_{1}$ and $X_{2}$ denote interference power in $N$ reference cells and jamming power in $M$ reference cells, respectively. $X_{1}$ is the summation of $N$ independent, exponential random variable (all with the same parameter $\lambda_{1}$ ); therefore, it obeys Erlang distribution [25], namely,

$$
f_{X_{1}}(x)= \begin{cases}\frac{\lambda_{1}^{N} x^{N-1}}{(N-1) !} e^{-\lambda_{1} x}, & x \geq 0, \\ 0, & x<0,\end{cases}
$$

where the parameter $\lambda_{1}=1 / \sigma_{i}^{2}$ is the inverse of average interference power in each cell. Similarly, the PDF of summation of $M$ false targets with mean power of $1 / \lambda_{2}=\sigma_{j}^{2}$ is

$$
f_{X_{2}}(x)= \begin{cases}\frac{\lambda_{2}^{M} x^{M-1}}{(M-1) !} e^{-\lambda_{2} x}, & x \geq 0, \\ 0, & x<0 .\end{cases}
$$

The PDF of total power in reference cells, denoted as $X_{3}=X_{1}+X_{2}$, is the convolution of $f_{X_{1}}(x)$ and $f_{X_{2}}(x)$,

$$
\begin{aligned}
f_{X_{3}}(x) & =f_{X_{1}}(x) * f_{X_{2}}(x) \\
& =\int_{0}^{x} \frac{\lambda_{1}^{N}(x-y)^{N-1}}{(N-1) !} e^{-\lambda_{1}(x-y)} \frac{\lambda_{2}^{M} y^{M-1}}{(M-1) !} e^{-\lambda_{2} y} \mathrm{~d} y \\
& =\frac{\lambda_{1}^{N} \lambda_{2}^{M} e^{-\lambda_{1} x}}{\Gamma(N) \Gamma(M)} x^{N+M-1} \times \int_{0}^{x}\left(1-\frac{y}{x}\right)^{N-1}\left(\frac{y}{x}\right)^{M-1} e^{\left(\lambda_{1}-\lambda_{2}\right) y} \mathrm{~d}\left(\frac{y}{x}\right) \\
& =\frac{\lambda_{1}^{N} \lambda_{2}^{M} e^{-\lambda_{1} x}}{\Gamma(N) \Gamma(M)} x^{N+M-1} \int_{0}^{1}(1-u)^{N-1} u^{M-1} e^{\lambda x u} \mathrm{~d} u,
\end{aligned}
$$

where $(*)$ denotes the convolution operator, and $\Gamma(\cdot)$ is the Gamma function. In the last line, we let $u=y / x$ and $\lambda=\lambda_{1}-\lambda_{2}$.

Since

$$
e^{\lambda x u}=\sum_{i=0}^{\infty} \frac{(\lambda x u)^{i}}{i !}
$$

equation (A.4) can be turned to

$$
\begin{aligned}
f_{X_{3}}(x) & =\frac{\lambda_{1}^{N} \lambda_{2}^{M} e^{-\lambda_{1} x}}{\Gamma(N) \Gamma(M)} \sum_{i=0}^{\infty} x^{N+M-1+i} \frac{\lambda^{i}}{i !} \int_{0}^{1}(1-u)^{N-1} u^{M-1+i} \mathrm{~d} u \\
& =\frac{\lambda_{1}^{N} \lambda_{2}^{M} e^{-\lambda_{1} x}}{\Gamma(N) \Gamma(M)} \sum_{i=0}^{\infty} x^{N+M-1+i} \frac{\lambda^{i}}{i !} \frac{\Gamma(M+i) \Gamma(N)}{\Gamma(M+N+i)} \\
& =\frac{\lambda_{1}^{N} \lambda_{2}^{M} e^{-\lambda_{1} x}}{\Gamma(M)} \sum_{i=0}^{\infty} x^{N+M-1+i} \frac{\lambda^{i}}{i !} \frac{\Gamma(M+i)}{\Gamma(M+N+i)} .
\end{aligned}
$$


The $2^{\text {nd }}$ equation in (A.6) involves the result of beta function, which implies

$$
B(p, q)=\int_{0}^{1}(1-u)^{p-1} u^{q-1} \mathrm{~d} u=\frac{\Gamma(p) \Gamma(q)}{\Gamma(p+q)} .
$$
derive

Substituting equations (12) and (A.6) into (10), we can

$$
\begin{aligned}
\overline{P_{\mathrm{D}}}(\gamma) & =\int_{0}^{\infty} P_{\mathrm{D}}(\gamma \mid x) f_{X_{3}}(x) \mathrm{d} x \\
& =\int_{0}^{\infty} \frac{\lambda_{1}^{N} \lambda_{2}^{M} e^{-\lambda_{1} x}}{\Gamma(M)} \sum_{i=0}^{\infty} x^{N+M-1+i} \frac{\lambda^{i}}{i !} \frac{\Gamma(M+i)}{\Gamma(M+N+i)} \cdot e^{-\gamma x} \mathrm{~d} x \\
& =\frac{\lambda_{1}^{N} \lambda_{2}^{M}}{\Gamma(M)} \sum_{i=0}^{\infty} \frac{\lambda^{i}}{i !} \frac{\Gamma(M+i)}{\Gamma(M+N+i)} \int_{0}^{\infty} e^{-k \lambda_{1} x} x^{N+M-1+i} \mathrm{~d} x \\
& =\frac{\lambda_{1}^{N} \lambda_{2}^{M}}{\Gamma(M)} \sum_{i=0}^{\infty} \frac{\lambda^{i}}{i !} \frac{\Gamma(M+i)}{\Gamma(M+N+i)} \cdot \frac{(M+N+i-1) !}{\left(k \lambda_{1}\right)^{M+N+i}} \\
& =\frac{1}{k^{M+N}} \sum_{i=0}^{\infty} \frac{1}{i !} \frac{\Gamma(M+i)}{\Gamma(M)}\left(\frac{\lambda_{1}}{\lambda_{1}}\right)^{N}\left(\frac{\lambda_{2}}{\lambda_{1}}\right)^{M}\left(\frac{\lambda_{1}-\lambda_{2}}{k \lambda_{1}}\right)^{i} \\
& =\frac{\theta^{M}}{k^{M+N}} \sum_{i=0}^{\infty} \frac{1}{i !} \frac{\Gamma(M+i)}{\Gamma(M)}\left(\frac{1-\theta}{k}\right)^{i} .
\end{aligned}
$$

In the $3^{\text {rd }}$ equation of (A.7), we define $k$ as

$\lambda_{1}+\gamma=\lambda_{1}+\alpha\left[\sigma_{i}^{2}(Q+\eta)\right]^{-1}=\left(1+\frac{\alpha}{N(Q+\eta)}\right) \lambda_{1} \triangleq k \lambda_{1}$.

The $4^{\text {th }}$ equation of (A.8) is due to the fact that

$$
\int_{0}^{\infty} e^{-a x} x^{n} \mathrm{~d} x=\frac{n !}{a^{n+1}} .
$$

And in the last line of (A.8), $\theta$ signifies the inverse of JIR and is defined as

$$
\theta \triangleq \frac{\lambda_{2}}{\lambda_{1}}=\frac{\sigma_{i}^{2}}{\sigma_{j}^{2}}=\frac{1}{\mathrm{JIR}}
$$

Since the fact that

$\sum_{i=0}^{\infty} \frac{1}{i !} \frac{\Gamma(M+i)}{\Gamma(M)} v^{i}=\sum_{i=0}^{\infty} \frac{1}{i !} \frac{(1)_{M+i}}{(1)_{M}} v^{i}=\sum_{i=0}^{\infty} \frac{(1+M)_{i}}{i !} v^{i}=(1-v)^{-M}$,

where $(\cdot)_{M}$ signifies rising factorials in the Pochhammer symbol, and the last equation in (A.12) is due to generalized binomial theorem. The final closed form of (A.8) can be expressed as

$$
\begin{aligned}
\overline{P_{\mathrm{D}}} & =\frac{\theta^{M}}{k^{M+N}}\left(1-\frac{1-\theta}{k}\right)^{-M}=k^{-N}\left(\frac{k-1+\theta}{\theta}\right)^{-M} \\
& =\left(1+\frac{\alpha}{N(Q+\eta)}\right)^{-N}\left(1+\frac{1}{\theta} \frac{\alpha}{N(Q+\eta)}\right)^{-M} .
\end{aligned}
$$

QED.

\section{Data Availability}

The data used to support the findings of this study are included within the article.

\section{Conflicts of Interest}

The authors declare that they have no conflicts of interest.

\section{References}

[1] M. A. Richards, J. A. Scheer, and W. A. Holm, Principles of Modern Radar Vol.1-Basic Principles, pp. 507-509, IET, London, UK, 2010.

[2] V. Hansen and J. Sawyers, "Detectability loss due to "greatest of" selection in a cell-averaging CFAR," IEEE Transactions on Aerospace and Electronic Systems, vol. 16, no. 1, pp. 115-118, 1980.

[3] M. Weiss, "Analysis of some modified cell-averaging CFAR processors in multiple-target situations," IEEE Transactions on Aerospace and Electronic Systems, vol. 18, no. 1, pp. 102$114,1982$.

[4] P. P. Gandhi and S. A. Kassam, "Analysis of CFAR processors in nonhomogeneous background," IEEE Transactions on Aerospace and Electronic Systems, vol. 24, no. 4, 1988.

[5] G. V. Trunk, "Range resolution of targets using automatic detectors," IEEE Transactions on Aerospace and Electronic Systems, vol. 14, no. 5, 1978.

[6] J. T. Rickard and G. M. Dillard, "Adaptive detection algorithms for multiple-target situations," IEEE Transactions on Aerospace and Electronic Systems, vol. 13, no. 4, 1977.

[7] J. Ritcey, "Performance analysis of the censored mean-level detector," IEEE Transactions on Aerospace and Electronic Systems, vol. 22, no. 4, pp. 443-454, 1986.

[8] H. Rohling, "Radar CFAR thresholding in clutter and multiple target situations," IEEE Transactions on Aerospace and Electronic Systems, vol. 19, no. 4, pp. 608-621, 1983.

[9] H. Rohling, "New CFAR-processor based on an ordered statistic," in Proceedings of the IEEE International Radar Conference, Arlington, June 1985.

[10] N. Tai, Y. Pan, and N. Yuan, "Quasi-coherent noise jamming to LFM radar based on pseudo-random sequence phasemodulation," Radio Engineering, vol. 4, 2016.

[11] Q. Jiang, Network Radar Countermeasure Systems Integrating Radar and Radar Countermeasures, Springer, Berlin, Germany, 2015.

[12] J. Tegler, "The next generation jammer (NGJ) and distributed electronic warfare," 2011, https://www.defensemedianetwork. $\mathrm{com} /$ stories/the-next-generation-jammer-and-distributed-ele ctronic-warfare.

[13] B. Clark, M. Gumzinger, and J. Sloman, Winning in the Gray Zone--Using Electromagnetic Warfare to Regain Escalation 
Dominance, Center for Strategic and Budgetary Assessments, Washington, D.C., USA, 2017.

[14] C. J. Watson, A Comparison of DDS and DRFM Techniques in the Generation of "Smart Noise" Jamming Waveforms, N. P. School, Monterey, CA, USA, 1996.

[15] Z. Yanbin, "Technology of smart noise jamming based on multiplication modulation," in Proceedings of the presented at the 2011 International Conference on Electric Information and Control Engineering, Wuhan, China, April 2011.

[16] M. B. El Mashade, "Analysis of the censored-mean level CFAR processor in multiple target and nonuniform clutter," IEE Proceedings-Radar, Sonar and Navigation, vol. 142, no. 5, pp. 259-266, 1995.

[17] M. B. El Mashade, "M-Sweeps detection analysis of cell-averaging CFAR processors in multiple-target situations," IEE Proceedings-Radar, Sonar and Navigation, vol. 141, no. 2, pp. 103-108, 1994.

[18] Y. Yan-Juan, Z. Feng, A. Xiao-Feng, L. Xiao-Bin, and $\mathrm{X}$. Zhong-Fu, "A study on effectiveness modeling of multiFalseTarget jamming," in Proceedings of the 2016 CIE International Conference on Radar 2016, Guangzhou, China, 2016.

[19] D. J. Bachmann, R. J. Evans, and B. Moran, "Game theoretic analysis of adaptive radar jamming," IEEE Transactions on Aerospace and Electronic Systems, vol. 47, no. 2, pp. 10811100, 2011.

[20] X. Liu and D. Li, "Analysis of cooperative jamming against pulse compression radar based on CFAR," EURASIP Journal on Applied Signal Processing, vol. 69, pp. 1-12, 2018.

[21] S. Kunpeng, F. Dejun, L. Yuan, and L. Lei, "The effect of intermittent sampling and repeated forwarding on the performance of radar constant false alarm rate detection," Aerospace Electronic Warfare, vol. 2, pp. 38-43, 2019.

[22] D. Ciuonzo and P. Salvo Rossi, "DECHADE: DEtecting slight changes with HArd DEcisions in wireless sensor networks," International Journal of General Systems, vol. 47, no. 5, pp. 535-548, 2018.

[23] D. Ciuonzo, A. De Maio, and P. Salvo Rossi, "A systematic framework for composite hypothesis testing of independent Bernoulli trials," IEEE Signal Processing Letters, vol. 22, no. 9, pp. 1249-1253, 2015.

[24] F. Gini, F. Lombardini, and L. Verrazzani, "Decentralized CFAR detection with binary integration in Weibull clutter," IEEE Transactions on Aerospace and Electronic Systems, vol. 33, no. 2, pp. 396-407, 1997.

[25] M. A. Richards, Fundamentals of Radar Signal Processing, McGraw-Hill Education, New York, NY, USA, 2nd edition, 2014. 